\title{
Conocimientos, actitudes y prácticas de padres/ cuidadores sobre salud bucal en el distrito de Ventanilla
}

Knowledge, attitudes and practices of parents / caregivers on oral health in the district of Ventanilla.

\author{
Jhoana Victorio- Pérez ${ }^{1, a}$, Wilfredo Mormontoy- Laurel ${ }^{3, d, e, f}$, María Elena Díaz-Pizán 2,a,b,c,d
}

\section{RESUMEN}

Objetivos: Determinar la relación entre los conocimientos, actitudes y prácticas de padres/cuidadores y el estado de salud bucal de sus niños de 3 a 5 años de edad pertenecientes al Centro Educativo Inicial (C.E.I) N $^{\circ} 120$ "Casuarinas", Distrito de Ventanilla. Material y Métodos: El estudio fue de tipo transversal y observacional. Se contó con la participación de 79 niños de 3 a 5 años de edad y sus respectivos padres/cuidadores. El análisis univariado de las variables cuantitativas se realizó mediante el cálculo de la media y desviación estándar (medidas de resumen), y de las variables cualitativas mediante la determinación de proporciones. La evaluación de la higiene bucal fue realizada según Green y Vermillion mediante el índice de higiene oral simplificado (IHO-S) y de caries dental según Gruebbel (índice ceo-d). Además, se evaluó la relación entre conocimientos, actitudes y prácticas (CAP) y caries dental e higiene bucal mediante el coeficiente de correlación de Spearman. Resultados: Se encontró una prevalencia de caries de infancia temprana (CIT) de 82,3\%, un ceo-d de 5,24 y un IHO-S de 1,56 en los niños evaluados. No se encontró asociación estadísticamente significativa entre los índices ceo-d e IHO-S de los niños y conocimientos, actitudes y prácticas sobre salud bucal de sus padres/cuidadores, excepto en actitud. Conclusiones: Se encontró un nivel regular de conocimientos, actitudes y prácticas, y una relación moderada estadísticamente significativa entre actitudes de los padres/cuidadores y el estado de salud bucal de sus niños (Índice ceo-d, p < 0,05; IHO-S, p < 0,05).

PALABRAS CLAVE: Caries dental, índice de higiene oral, conocimientos, actitudes, prácticas en salud, salud bucal. 


\section{SUMMARY}

Objectives: To determine the relationship between the knowledge, attitudes and practices of parents / caregivers and the oral health status of their children from 3 to 5 years of age belonging to the Initial Educational Center (CEI) N ${ }^{\circ} 120$ "Casuarinas", Ventanilla District. Material and Methods: The study was cross-sectional and observational. It was attended by 79 children from 3 to 5 years of age and their respective parents / caregivers. The univariate analysis of the quantitative variables was performed by calculating the mean and standard deviation (summary measures), and qualitative variables by determining proportions. The evaluation of oral hygiene was carried out according to Green and Vermillion using the simplified oral hygiene index (IHO-S) and dental caries according to Gruebbel (ceo-d index). In addition, the relationship between knowledge, attitudes and practices (CAP) and dental caries and oral hygiene was assessed using the Spearman correlation coefficient. Results: A prevalence of early childhood caries (EEC) of $82.3 \%$, a ceo-d of 5.24 and an IHO-S of 1.56 was found in the children evaluated. No statistically significant association was found between the ceo-d and IHO-S indices of the children and the knowledge, attitudes and practices on oral health of their parents / caregivers, except in attitude. Conclusions: We found a regular level of knowledge, attitudes and practices, and a statistically significant moderate relationship between attitudes of parents / caregivers and the oral health status of their children (ceo-d index, $\mathrm{p}<0.05$; IHO-S, $\mathrm{p}<0.05$ ).

\section{KEY WORDS: Dental caries, index of oral hygiene, knowledge, attitudes, practices in health, oral health.}

\section{INTRODUCCIÓN}

La caries dental, enfermedad periodontal y las maloclusiones representan grandes retos para los odontólogos por su multicausalidad. Su prevención depende de varios factores entre los que se encuentran: la presencia de flúor, las posibilidades de acceso a los centros de salud entre otros; y principalmente depende de los conocimientos, actitudes y prácticas de los padres/cuidadores sobre la salud bucal (1).

La caries de infancia temprana (CIT) es una de las patologías más frecuentes en la niñez cuya etiología es multifactorial. Son importantes los conocimientos, prácticas y actitudes de los padres/cuidadores en su prevención y control, así como también los hábitos de alimentación, cuidados en la higiene bucal, frecuencia de visitas al odontólogo, presencia y severidad de la enfermedad, etc. Para la prevención de la CIT es necesario elaborar programas de promoción, educación y prevención, adecuados para cada comunidad, mejorando así los niveles epidemiológicos de la enfermedad (2).

El año 2006 Delgado-Angulo et al., en la comunidad urbano-marginal Sol Naciente (Carabayllo), evaluaron los cambios en los conocimientos en salud bucal de un grupo de padres/cuidadores después de un programa educativo de 18 meses de intervención (char- las educativas) (3). Los resultados fueron positivos, encontrándose mejoras en los conocimientos en los ítems de alimentación, cepillado dental, uso del flúor y una temprana visita del niño al odontólogo. Respecto a las actitudes de los padres/cuidadores sobre salud bucal, los autores observaron una mejora estadísticamente significativa luego de la intervención. Sobre prácticas en salud bucal, encontraron una diferencia estadísticamente significativa antes y después de la intervención en todos los tópicos evaluados, menos en el ítem de consumo de alimentos azucarados entre comidas y el cepillado nocturno.

Así también, el año 2012 Benavente et al., publicaron una investigación realizada en centros hospitalarios del Ministerio de Salud (MINSA) y del Seguro Social de Salud (ESSALUD) cuyo objetivo fue determinar cuánto conocían las madres de familia sobre el estado de salud bucal de sus niños menores de cinco años de edad. Participaron 143 madres, encontrándose que no existió asociación entre el nivel de conocimientos en salud bucal y el estado bucal de sus niños; siendo éste conocimiento regular, observándose en los niños un índice de caries dental alto (ceo-d= 4.5 a 6.5) (4).

El año 2015, Cupé-Araujo y García-Rupaya realizaron un estudio en cuatro Instituciones Educativas Públicas de los distritos de Lima Metropolitana, donde evaluaron el nivel de conocimientos de 312 padres y/o madres de familia, encontrándose que es- 
tos sí poseían un nivel de conocimiento bueno sobre salud bucal (2).

Estos resultados en algunos casos opuestos y divergentes justificaron el profundizar y estudiar el mismo tema en otras poblaciones. Además, es en la primera infancia y etapa preescolar cuando se instauran los hábitos saludables en el individuo. Padres con conocimientos y prácticas buenas y con actitudes adecuadas tendrán niños con buena salud bucal (5).

El propósito del presente estudio fue determinar la relación entre los conocimientos, actitudes y prácticas de padres/cuidadores y el estado de salud bucal de sus niños de 3 a 5 años de edad, del Centro Educativo Inicial N ${ }^{\circ} 120$ "Casuarinas", Distrito de Ventanilla.

\section{MATERIAL Y MÉTODOS}

El presente estudio fue de tipo transversal, descriptivo. Las variables que se midieron fueron: a) En el padre/cuidador: conocimientos, actitudes y prácticas sobre la salud bucal de sus niños; b) En el niño: caries dental e higiene bucal. Las covariables evaluadas fueron: a) En el padre/cuidador: edad, sexo y nivel de instrucción, b) En el niño: edad y sexo.

Las técnicas que se emplearon fueron la encuesta y la observación. Mediante la encuesta se midieron conocimientos, prácticas y actitudes sobre salud bucal de los padres/cuidadores de los niños de la muestra y mediante la observación se recogieron datos de los índices clínicos ceo-d para caries dental e IHO-S para higiene bucal. En la ficha clínica se registraron los datos del padre/ cuidador y del niño sobre: edad, sexo y nivel de instrucción del padre/cuidador y del niño según correspondió.

Se empleó una encuesta, validada y aplicada como parte del trabajo de investigación: "Conocimientos, actitudes y prácticas de madres de familia sobre salud oral en preescolares, en el policlínico Pacasmayo ESSALUD, 2014". En la validación de la encuesta se empleó la prueba alfa de Cronbach, siendo los resultados: para conocimiento sobre salud bucal $\alpha=0,76$, para actitudes $\alpha=0,80$ y para prácticas $\alpha=0,72$ (6).
La calibración del investigador principal del presente estudio para la obtención de los datos clínicos ceo-d e IHO-S fue efectuada con el apoyo de un docente especialista en odontopediatría con más de 10 años de experiencia. Empleándose la clasificación de Green y Vermillion para higiene bucal y Gruebbel para caries dental (7-10). Se empleó la Escala de Estanino para categorizar los puntajes de las variables conocimientos y prácticas sobre salud bucal, agrupándose en tres categorías, sobre las bases de las medias y las desviaciones estándar. Los puntajes de la variable actitud sobre salud bucal fueron divididos en dos categorías usando como punto de corte, el promedio.

Se obtuvo la aprobación del Comité Institucional de Ética de la Universidad Peruana Cayetano Heredia (UPCH), así como las autorizaciones respectivas de las autoridades educativas para la ejecución del estudio. La población estuvo conformada por 150 niños, quienes asistían regularmente al Centro Educativo "Casuarinas". De los cuales sólo 79 aceptaron formar parte de la investigación, debido a que los padres/cuidadores del resto de los niños trabajaban todo el día.

Los criterios de selección para formar parte del estudio fueron: a) niños de 3 a 5 años de edad, que presentaron dentición primaria completa $\mathrm{y}$ fueron receptivos al examen clínico; y b) padres/ cuidadores que otorgaron su consentimiento para el estudio, y que permanecían más de doce horas diarias con el niño. La recolección de datos se realizó en dos etapas, primero se entrevistó a los padres/cuidadores y finalmente se realizó el examen clínico bucal de los niños.

Se organizó una reunión con los padres/cuidadores, en las instalaciones del centro educativo, donde se expuso el objetivo de la investigación y los procedimientos que se llevarían a cabo para la realización del estudio. Una vez obtenido el consentimiento informado, se les interrogó acerca de sus conocimientos, actitudes y prácticas sobre salud bucal respecto a sus niños. El asentimiento fue dado por los niños de la muestra que podían hacerlo. Se les explicó de una manera sencilla en qué consistiría el examen bucal y 
finalmente los padres/cuidadores y los niños de mayor edad (5 años de edad) recibieron una charla sobre higiene bucal al finalizar el examen clínico. La evaluación clínica bucal fue realizada en el local de la institución, a plena luz del día, posicionando a los niños en una silla y con el uso de luz natural. El examinador se ubicó detrás del niño(a) para la revisión de la cavidad bucal. El examen clínico se realizó con un espejo plano sin aumento y la sonda de exploración de la Organización Mundial de la Salud (OMS).

La base de datos fue confeccionada y almacenada en un ordenador haciendo uso del programa Microsoft Excel 2010. Para el análisis estadístico se empleó el programa estadístico Stata 15, con licencia en la Universidad Peruana Cayetano Heredia. Se calcularon las medidas de resumen para las variables cuantitativas: media y desviación estándar y para las variables cualitativas: proporciones (\%). Además, se calcularon las medidas de asociación bivariada: $\mathrm{r}$ de Spearman para las variables cuantitativas (conocimiento, prácticas y actitudes de los padres/cuidadores con edad, sexo y nivel de instrucción de ellos y con caries dental e higiene bucal de los niños. Para la presentación de los resultados se usó tablas bivariados.

\section{RESULTADOS}

La mayoría de los padres/cuidadores que respondieron la encuesta fueron de sexo femenino $(84,8 \%)$, de 20 a 29 años de edad $(51,9 \%)$, con edad promedio de 32 años $( \pm 8,5)$ y grado de instrucción secundaria $(49,4 \%)$; solo un padre/cuidador fue analfabeto $(1,2 \%)$ (Tabla 1$)$.

El $57 \%$ de los niños fueron del sexo masculino. La frecuencia de los niños aumentó conforme aumento la edad (3años $=16,4 \%, 4$ años $=26,6 \%, 5$ años $=57 \%$ ). $\mathrm{Al}$ evaluar el IHO-S se encontró un promedio regular, equivalente a 5,2 $( \pm 0,6)$, observándose a 7 niños con un nivel de higiene bueno $(8,9 \%), 71$ niños con un nivel regular $(89,9 \%)$ y solo un niño con un nivel malo $(1,2 \%)$. El índice promedio de ceo-d fue muy alto, equivalente a $5,2( \pm 4,2)$, encontrándose ser muy bajo en 20 niños $(25,3 \%)$, bajo en 8 niños $(10,1 \%)$, modera$d o$ en 9 niños $(11,4 \%)$ y alto en $42(53,2 \%)$ (Tabla 1$)$.
Existió una baja relación directa entre conocimiento de los padres/cuidadores y el estado de salud bucal (ceo-d e IHO-S) de sus niños $\left(r_{\mathrm{s}}=0,18 ; r_{\mathrm{s}}=0,15\right)$; es decir, a mayor conocimientos sobre salud bucal, mayor fue el índice ceo-d e IHO-S, pero los datos presentaron una gran dispersión. No siendo la asociación estadísticamente significativa (Tabla 2). Respecto a los conocimientos de los padres/cuidadores sobre alimentación, el 77,7\% respondió que el cambio de biberón a taza debería iniciarse a partir de los 15 meses; y sobre el principal tipo de comida que puede causar caries dental, los padres/cuidadores respondieron en su mayoría (65\%) que era el azúcar. En lo referente a tratamiento dental, la mayoría $(63,3 \%)$ de los padres/cuidadores respondieron que no era necesario restaurar los dientes afectados por caries dental ya que se iban a caer (exfoliar).

Existió relación moderada directa entre actitudes de los padres/cuidadores y el estado de salud bucal (ceo-d e IHO-S) de sus niños $\left(r_{s}=0,26 ; r_{s}=0,35\right)$; es decir, a mejor actitud del padre/cuidador sobre salud bucal, mayor fue el índice ceo-d e IHO-S, existiendo una dispersión de los datos más cercana a 1 , la asociación fue estadísticamente significativa (tabla 2). En lo referente a actitud sobre el uso del biberón y si su uso prolongado puede producir mala posición de dientes, los padres/cuidadores en su mayoría $(84,1 \%)$ dijeron que era mejor que los niños dejen el biberón a los 15 meses de edad para evitar mal posiciones dentarias o hábitos.

Existió relación baja indirecta entre prácticas de los padres/cuidadores y el estado de salud bucal (ceo-d e IHO-S) de sus hijos $\left(r_{\mathrm{s}}=-0,12 ; \mathrm{r}_{\mathrm{s}}=0,16\right)$; es decir, a mejores prácticas, menor fue el índice ceo-d e IHO-S, existiendo una gran dispersión de datos. Sin embargo, la asociación no fue estadísticamente significativa (Tabla 2). Específicamente, respecto a prácticas sobre higiene bucal, el $73,6 \%$ de los padres/ cuidadores respondieron que realizaban el cepillado de los dientes de sus niños 3 veces al día, y el 88,5\% respondieron que no conocían la cantidad de pasta dental que debían colocar en el cepillo para hacerle la higiene bucal de su niño. 
Tabla 1. Características sociodemográficas y clínicas de los padres/cuidadores y de sus niños de 3 a 5 años de edad, Centro Educativo Inicial N 120 "Casuarinas", Distrito de Ventanilla.

\begin{tabular}{|c|c|c|}
\hline $\begin{array}{l}\text { Características } \\
\text { sociodemográficas y } \\
\text { clínicas }\end{array}$ & $\mathrm{n}=\mathbf{7 9}$ & $\%$ \\
\hline \multicolumn{3}{|l|}{ Padres/cuidadores } \\
\hline \multicolumn{3}{|l|}{ Sexo } \\
\hline Femenino & 67 & 84,8 \\
\hline Masculino & 12 & 15,2 \\
\hline \multicolumn{3}{|l|}{ Edad (años) } \\
\hline 20 a 29 & 41 & 51,9 \\
\hline 30 a 39 & 23 & 29,1 \\
\hline$\geq 40$ & 15 & 19,0 \\
\hline \multicolumn{3}{|c|}{$(\overline{\mathrm{x}}=32 ; \mathrm{DE} \pm 8,5)$} \\
\hline \multicolumn{3}{|l|}{ Grado de instrucción } \\
\hline Analfabeta & 1 & 1,2 \\
\hline Primaria & 16 & 20,3 \\
\hline Secundaria & 39 & 49,4 \\
\hline Técnico & 20 & 25,3 \\
\hline Superior & 3 & 3,8 \\
\hline \multicolumn{3}{|l|}{$\underline{\text { Del niño }}$} \\
\hline \multicolumn{3}{|l|}{ Sexo } \\
\hline Femenino & 34 & 43,0 \\
\hline Masculino & 45 & 57,0 \\
\hline \multicolumn{3}{|l|}{ Edad (años) } \\
\hline 3 años & 13 & 16,4 \\
\hline 4 años & 21 & 26,6 \\
\hline 5 años & 45 & 57,0 \\
\hline \multicolumn{3}{|c|}{$(\overline{\mathrm{x}}=4,5 ; \mathrm{DE} \pm 0,9)$} \\
\hline \multicolumn{3}{|l|}{ IHO-S* } \\
\hline Bueno $(0,0$ a 1,2$)$ & 13 & 8,9 \\
\hline Regular $(1,3$ a 3,0$)$ & 71 & 89,9 \\
\hline Malo $(3,1$ a 6,0$)$ & 1 & 1,2 \\
\hline \multicolumn{3}{|c|}{$(\overline{\mathrm{x}}=5,2 ; \mathrm{DE} \pm 0,6)$} \\
\hline \multicolumn{3}{|l|}{ ceo-d* } \\
\hline Muy bajo $(0,1$ a 1,1$)$ & 20 & 25,3 \\
\hline Bajo $(1,2$ a 2,6$)$ & 8 & 10,1 \\
\hline Moderado $(2,7$ a 4,4$)$ & 9 & 11,4 \\
\hline Alto $(4,5$ a 6,5$)$ & 42 & 53,2 \\
\hline \multicolumn{3}{|c|}{$(\overline{\mathrm{x}}=5,2 ; \mathrm{DE} \pm 4,2)$} \\
\hline $\begin{array}{l}\overline{\mathrm{x}}: \text { media } \\
\text { DE }: \text { Desviación Estandar } \\
* \text { IHO-S } \\
\text { *ceo-d }^{13,31,31,32}: \geq 1=79(10 \\
\text { Niños sano= }\end{array}$ & 80 & \\
\hline
\end{tabular}


Tabla 2. Conocimientos, actitudes y prácticas sobre salud bucal de padres/cuidadores relacionados a los índices ceo-d e IHO-S de sus niños de 3 a 5 años de edad, Centro Educativo Inicial $N^{\circ} 120$ "Casuarinas", Distrito de Ventanilla.

\begin{tabular}{|c|c|c|c|c|c|c|c|c|c|}
\hline & \multicolumn{4}{|c|}{ Índice ceo-d } & \multicolumn{3}{|c|}{ Índice IHO-S } & \multirow{3}{*}{ Total } & \multirow{3}{*}{$\%$} \\
\hline & $\begin{array}{l}\text { Muy } \\
\text { Bajo }\end{array}$ & Bajo & Moderado & Alto & Bajo & Moderado & Alto & & \\
\hline \multicolumn{8}{|l|}{ Variables } & & \\
\hline \multicolumn{10}{|l|}{ Conocimiento } \\
\hline Bueno (18-20) & 1 & 2 & 0 & 6 & 1 & 7 & 1 & 9 & 11,4 \\
\hline Regular (12-17) & 13 & 3 & 7 & 30 & 2 & 51 & 0 & 53 & 67,0 \\
\hline Mala (0-11) & 6 & 3 & 2 & 6 & 4 & 13 & 0 & 17 & 21,0 \\
\hline$\underline{\text { Actitud }}^{r_{s=0,18203}}$ & \multicolumn{4}{|c|}{$\mathrm{p}=0,10836$} & \multicolumn{3}{|c|}{$r_{s=0,15284} \quad p=0,1787$} & & \\
\hline Adecuada (23-27) & 6 & 2 & 6 & 18 & 1 & 30 & 1 & 32 & 40,0 \\
\hline Inadecuada (9-22) & 14 & 6 & 3 & 24 & 6 & 41 & 0 & 47 & 59,0 \\
\hline \multicolumn{3}{|l|}{ Práctica } & & & \multicolumn{3}{|c|}{$r_{s=0,34805 \quad p=0,00167}$} & & \\
\hline Buena (28-40) & 5 & 3 & 1 & 5 & 4 & 10 & 0 & 14 & 17,8 \\
\hline Regular (23-27) & 8 & 5 & 8 & 27 & 1 & 45 & 1 & 47 & 59,0 \\
\hline Mala (10-22) & 7 & 0 & 2 & 8 & 1 & 17 & 0 & 18 & 22,8 \\
\hline$r_{s=-0,11575}$ & \multicolumn{4}{|c|}{$\mathrm{p}=0,03097$} & \multicolumn{3}{|c|}{$\mathrm{r}_{\mathrm{s}=-0,15839} \quad \mathrm{p}=0,16326$} & & \\
\hline
\end{tabular}

\section{DISCUSIÓN}

La muestra del presente estudio $(\mathrm{n}=79)$ fue menor a los estudios realizados por Franco (11) en Colombia 1995 (n=296) (11), Martignon el año $2003(\mathrm{n}=770)$ (12) y Franco et al., el año $2004(n=365)(13)$; en E.E.U.U por Hardison et al., el año $2003(n=572)$ (14); en Perú por Benavente et al., el 2012 ( $n=143)$ (4), Cupe-Araujo y Grarcia-Rupaya el $2015(n=190)$ (2). Pero, similar a la muestra de los estudios peruanos de Ávalos-Márquez et al., quienes estudiaron a un grupo de escolares $(n=76)$ de la I.E 1120 "Pedro A. Labarthe" en Ventanilla el año 2012 (5), y Suyo y Iannnacone $(\mathrm{n}=58)$ que evaluaron un grupo de niños preescolares del Centro de Salud de Mala el año 2009
(15). La razón del poco número de la muestra fue debido a que los padres/ cuidadores de los niños, no asistieron a las citaciones realizadas, lo hacían sólo si de por medio existía algún beneficio económico o material, tal como se comprobó en campañas anteriores.

La mayoría de los padres/cuidadores tuvieron entre 20 a 29 años de edad. Estos resultados coinciden con los estudio de Suyo et al., y Teixeira et al., donde más de la mitad de las madres que se encuestó no superaba los 29 años de edad $(15,16)$. Esto se puede explicar porque en el distrito de Ventanilla, existen más pobladores jóvenes (15 a 29 años), según el INEI (17). Respecto a la experiencia de caries dental, en la presente investigación, los valores que se obtuvieron en 
el índice ceo-d fueron altos $(5,24 \pm 4,21)$. Investigaciones a nivel mundial muestran índices ceo-d más bajos. Así, Prasai et al., al evaluar a un grupo de niños el año 2013 en Asia, obtuvieron un ceo-d bajo, equivalente a 1,59 (ceo-d bajo=1,2 a 2,6) y el año 2012 en Pakistan, Dawani et al., encontraron un ceo-d también bajo $(2,08)(18,19)$. La explicación de estos resultados estaría en que en estos países, el cuidado de la cavidad bucal es importante brindando el estado programas de prevención en los centros de salud.

A nivel nacional, Bravo el año 2014 encontró un índice ceo-d alto de 4,6 (ceo-d alto $=4,5$ a 6,5) (20). El año 2011, Villena-Sarmiento et al., al examinar un grupo de niños de 6 a 41 meses de edad en comunidades urbano marginales de Carabayllo encontraron un índice ceo-d moderado de 2,97 (ceo-d moderado $=2,7$ a 4,4) $(21)$. Los padres/cuidadores del centro educativo de Ventanilla evaluados en el presente estudio, a pesar que tuvieron conocimientos básicos sobre salud bucal, no aplicaron dichos conocimientos, actitudes y prácticas en el cuidado de la salud bucal de sus hijos. Tal vez por la poca motivación de la población a pesar de existencia de programas de prevención en salud bucal que ofrece la UPCH o por la falta de tiempo, debido a las tareas domésticas o al alto número de hijos que por lo general poseen.

Respecto a la higiene bucal, se obtuvo un valor regular en el índice IHO-S (IHO-S=1,3-3,0). Lo cual coinciden con los hallazgos de algunas investigaciones, como las de Bravo (2014) (IHO-S= 2,3) (20), y Villanueva-Velásquez et al., (IHO-S=1,36) (22). Sorprenden los resultados del presente estudio, ya que a pesar del tiempo que tiene el programa de educación y prevención de la UPCH (más de 3 años), se encontró un IHO-S bajo $(1,3)$; sin embargo, el índice ceo-d fue alto $(5,24)$. Esto se puede explicar porque la caries dental es una enfermedad multifactorial donde hay que tener en cuenta otros factores como ambientales, socioeconómicos, etc., $(23,24)$.

Respecto a los conocimientos, en el presente estudio se observó un nivel regular, encontrándose una baja relación directa, entre conocimientos de los padres/ cuidadores sobre salud bucal y las condiciones bucales de sus niños; es decir, a mayores conocimientos, mayores fueron los índices ceo-d e IHOS. Resultados similares fueron hallados por Gao et al., el año 2011, al evaluar 138 padres de niños de 3 a 5 años de edad al sur de China (25); por Wilson et al., el año 2016, al evaluar un grupo de cuidadores de niños americanos de 3 a 5 años de edad (nativos de la India/Alaska) (26) y por Wilson et al., el año 2017, al evaluar un grupo de 100 madres latinas residentes en E.E.U.U (27), encontrándose en todos los casos que a mayores conocimientos sobre salud bucal, sus niños presentaban mayores lesiones de caries dental y eran más susceptibles a desarrollar la enfermedad. Hooley et al., el año 2012, encontraron una relación estadísticamente significativa entre conocimientos de los padres y caries dental en sus niños, afirmando que los conocimientos, actitudes y prácticas de los padres, influyen en las decisiones que éstos toman con respecto a sus hijos; reflejándose también en su conducta, gustos, preferencias, etc., que serán desarrollados a lo largo de su vida (28). Estos resultados se pueden explicar por la falta de tiempo que tendrían los padres/cuidadores de tales niños, para dedicarse a su cuidado ya que la mayoría tienen más de 3 hijoS (29). Pues a pesar de tener buenos conocimientos sobre salud bucal; sin embargo, eso no se ve reflejado en el estado de salud bucal de sus niños, quienes presentan mayores lesiones de caries dental.

Estos resultados fueron inferiores a los encontrados por Cupé-Araujo y García-Rupaya (2) y a los de Delgado-Angulo et al., (3), donde el nivel de conocimiento de los padres fue bueno. Otras investigaciones como las de Teixeira et al., el año 2010 (Paraguay) (16), y Nobile et al., el año 2014 (Italia) (30), mostraron resultados inferiores a la presente investigación (bajo conocimiento). El nivel de conocimientos sobre salud bucal fue bajo, a pesar que son países con una mejor economía. Al evaluar algunos subtemas específicos respecto a conocimiento de los padres/cuidadores sobre salud bucal se encontraron: a) En lo referente a alimentación de los niños, la mayoría de padres/ cuidadores del presente estudio tuvieron conocimientos que una dieta balanceada era esencial y los alimentos para su consumo debían ser bajos en azúcares por ser un factor que predispone a la aparición de lesiones cariosas. Además, los padres/cuidadores alternaban refrescos del almuerzo a base de agua pura y evitaban ponerle azúcar a la leche de sus niños.

Este conocimiento fue recibido de los estudiantes de la UPCH, quienes tienen en la zona un programa de intervención educativo/preventivo que desarrollan en los colegios y casa por casa. b) Con respecto, al 
tratamiento dental, en el presente estudio la mayoría de los padres/cuidadores no conocían la importancia de mantener los dientes en boca. Similares resultados fueron encontrados por Franco et al., en Colombia en una población de 296 madres, donde la mayoría de ellas no le daban importancia a tener los dientes sanos o no restauraban los dientes de sus niños (11). La explicación estaría en la falta de tiempo, al temor de los niños de entrar a la consulta, al tiempo de espera demasiado largo de las citas y al difícil acceso a un centro de salud, entre otros.

Respecto a las actitudes sobre salud bucal, en el presente estudio se encontró una relación moderada directa estadísticamente significativa entre actitudes adecuadas de los padres y el índice ceo-d e IHO-S, es decir, a mejor actitud del padre/cuidador sobre salud bucal, mayor fue el índice ceo-d e IHO-S. Gao et al., (25) y Wilson et al., (26) no encontraron relación estadísticamente significativa entre ambas variables.

Esto, se pude explicar porque los padres a pesar de saber cómo cuidar la boca de sus niños no le dedican mucho tiempo a sus hijos ya que para ellos lo más importante es trabajar para ganar un poco de dinero y así cubrir los gastos del hogar. Además, los niños por lo general están al cuidado de una tercera persona que en la mayoría de los casos es la abuela (29).

Los estudios de Delgado-Angulo et al., el año 2006 (Perú) (3), y Martignon et al., el año 2008 (Colombia) (12), muestran resultados diferentes, encontrando que los padres presentaron actitudes favorables, en dichos estudios no se evaluó el estado de salud bucal del niño. Mientras que Villanueva-Velásquez et al., el año 2014 (Perú), al evaluar las actitudes de un grupo de padres encontraron que éstas fueron también bajas (22). Al evaluar actitudes respecto al uso del biberón, en el presente estudio, la mayoría de los padres/cuidadores respondieron que era mejor que el niño deje el biberón a los 15 meses de edad, porque su uso prolongado podía originar complicaciones con la posición de sus dientes o crearles hábitos nocivos. A diferencia de Rodríguez et al., en Cuba (23), quienes encontraron que los padres/cuidadores pensaban que no era necesario suspender el uso del biberón sino, que el niño debería dejarlo por sí solo. Al respecto, la literatura nos refiere que el biberón puede transformarse en un elemento negativo a la hora de dormir, aumentando la aparición de lesiones cariosas si es que no va acompañado de un cepillado adecuado por las noches $(23,24)$. Estos aspectos son conocidos por los padres/cuidadores del presente estudio a través de las sesiones educativas que vienen recibiendo durante varios años por los estudiantes de la UPCH y del personal de los centros de salud.

Al evaluar las prácticas sobre salud bucal, el presente estudio encontró que a mejores prácticas de los padres/cuidadores, menores fueron los índices ceo-d e IHOS; este resultado se debería a que los encuestados en su mayoría fueron madres con un nivel de educación secundaria, lo cual se traduce en buenas prácticas y buenos conocimientos sobre salud bucal. Resultados contrarios fueron hallados por Hooley et al., quienes al evaluar un grupo de 45 padres de niños europeos de 0 a 6 años de edad, encontraron que a mejores prácticas, mayor enfermedad bucal del niño (28).

Otro estudio realizado en nuestro país en el distrito de Carabayllo, se encontró las actitudes de los padres muy buenas (3). Al evaluar las prácticas de los padres/cuidadores referentes: a) La higiene bucal del niño en el presente estudio, los padres/cuidadores señalan la importancia del cuidado de la boca desde la etapa de recién nacido (antes de la erupción de los dientes), limpiando la boca de sus niños tres veces al día. Estos resultados coinciden con los investigadores Franco et al., en Colombia, quienes manifiestan resultados similares (11). b) En relación al tamaño de la pasta dental que deben usar los niños, en el presente estudio, se encontró que no siempre era la correcta y que los niños realizan su higiene bucal muchas veces sin supervisión de sus padres o de un adulto responsable. Esto difiere de lo encontrado por Martignon en Colombia, donde la mayoría de padres/cuidadores realizan la limpieza bucal a sus pequeños niños (12). c) En relación, a la visita al odontólogo, la mayoría de los padres/cuidadores del presente estudio manifestaron que no tenían como práctica el llevar a sus niños para un examen clínico temprano; a diferencia de otros padres de familia del estudio de Martignon et al., en Colombia en una muestra de 770 participantes (12).

En general, los resultados contrarios a lo esperado en el presente estudio, se explicarían porque los conocimientos, actitudes y prácticas de los padres/cuidadores no estarían influyendo en la disminución de los índices de CIT e higiene bucal de sus hijos, debido a que existirían otras variables que no han sido estudia- 
das y que influirían en los resultados.

En conclusión, en el presente estudio, no se encontró relación estadísticamente significativa entre conocimientos y prácticas de los padres/cuidadores y el estado de salud bucal (índice ceo-d e IHO-S) de sus hijos. Pero, si se encontró relación moderada estadísticamente significativa entre actitudes inadecuadas con el estado de salud bucal de los niños de 3 a 5 años de edad.

\section{Correspondencia:}

Jhoana Milagros Victorio Pérez

Correo electrónico: jhoana.victorio.p@upch.pe

\section{REFERENCIAS BIBLIOGRÁFICAS}

1. Rolón M, Samudio M. Conocimiento, actitud y práctica de los médicos pediatras sobre factores preventivos de la salud oral en la primera infancia. Pediatría (Asunción). 2014; 41(3):191 - 200.

2. Cupe-Araujo A, Garcia-Rupaya C. Conocimientos de los padres sobre la salud bucal de niños preescolares: desarrollo y validación de un instrumento. Rev Estomatol Herediana. 2015; 25(2): 112-121. DOI: https://doi.org/10.20453/reh.v25i2.2457

3. Delgado-Angulo E, Sánchez-Borjas PC, Bernabé E. Mejora en los conocimientos, actitudes y prácticas a través de una intervención en salud oral basada en comunidad. Rev Estomatol Herediana. 2006; 16 (2): 83-88. DOI: https://doi.org/10.20453/reh.v16i2.1898

4. Benavente L, Chein S, Campodónico C, et al. Nivel de conocimientos en salud bucal de las madres y su relación con el estado de salud bucal del niño menor de cinco años de edad. Odontología Sanmarquina 2012; 15(1): 14-18.

5. Ávalos-Márquez J, Huillca-Castillo N, Picasso-Pozo M, Ornori-Mitumori E, Gallardo-Schultz A. Nivel de conocimientos en salud oral relacionado con la calidad de la higiene oral en escolares de una población peruana. KIRU. 2015; 12(1):61-65.

6. Ríos C. Conocimientos, actitudes y prácticas de madres de familia sobre salud oral en preescolares, en el policlínico Pacasmayo ESSALUD, 2014. Tesis de Bachiller. Trujillo, Perú: Universidad Privada Antenor Orrego 2014. 52 pp.

7. Ministerio de Salud. Indicadores epidemiológicos para la caries dental. Lima, Perú: Ministerio de Salud; 2013.

8. Aguilar M, Canamas M, Ibáñez P, Gil F. Importancia del uso de índices en la práctica periodontal diaria del higienista dental. Periodoncia. 2003; 13(1): 233-244.

9. Newman M, Carranza F. C. Periodontología Clínica.
Ciudad de Mexico: Edit Mac Graw-Hill Interamericana; 1998.

10. Heredia C, Acosta J, Flores M, Gonzales B, Melgar R, Yamamoto F. Odontología Preventiva en el niño y en el adolescente, Manual de procedimientos clínicos. Lima: Universidad Peruana Cayetano Heredia; 2012.p. 1-53.

11. Franco A. Conocimientos y prácticas de salud oral de las madres de niños preescolares, Medellín, 1994. Revista CES Odontología. 1995; 8(2):132-135.

12. Martignon S, González M C, Jacome S, Velosa J, Santamaría R. Conocimientos, actitudes y prácticas en salud oral de padres y jardineras de niños hogares infantiles: ICBF - Usaquén, Bogotá. Revista de Salud Pública. 2003; 9 (2): 1-15.

13. Franco A, Santamaría A, Kurzer E, Castro L, Giraldo M. El menor de seis años: Situación de caries y conocimientos y prácticas de cuidado bucal de sus madres. Revista CES Odontología. 2004; 17(1): 19-29.

14. Hardison J, Cecil J, Mullins M, White J, Manz M, Ferretti G. The 2001 Kentucky Children's Oral Health Survey Findings for Children Ages 24 to 59 months and Their Caregivers. Pediatric Dentistry. 2003; 25(4): 365-371.

15. Suyo T, Iannnacone J. Influence of teaching about oral hygiene awareness to parents of children less than three year in the health center of Mala, Peru. The Biologist. 2013; 11(2):307-319.

16. Teixeira P, Vázquez C, Domínguez V, et al. Nivel de conocimiento de madres sobre higiene bucal en niños de 0 a 3 años. Hospital Materno Infantil San Pablo Paraguay, 2010. Revista Salud Pública Paraguay. 2011; $1(1): 3-12$.

17. Hidalgo N, Hidalgo N, Benavides H. Crecimiento económico, población, características sociales y seguridad ciudadana en la provincia constitucional del Callao. Lima: Instituto Nacional de Estadística e Informática; 2016.p.1-197.

18. Prasai L, Shakya A, Shrestha M, Shrestha A. Dental caries prevalence, oral heath knowledge and practice among indigenous Chepang school children of Nepal. BMC Oral Health. 2013; 13(20):1-5.

19. Dawani N, Nisar N, Khan N, Syed S, Tanweer N. Prevalence and factors related to dental caries among pre-school children of Saddar town, Karachi, Pakistan: a across-sectional study.BMC Oral Health. 2012; 12(59):1-9.

20. Bravo F. Asociación entre el grado de instrucción de la madre y sus conocimientos sobre salud bucal en el estado de salud bucal de sus hijos de 5-12 años de edad de la ciudadela Pachacútec, distrito de Ventanilla, Provincia del Callao en el año 2014. Tesis de Bachiller. Lima, Perú: Universidad Peruana Cayetano Heredia. 55 pp.

21. Villena-Sarmiento R, Pachas-Barrionuevo F, Sán- 
chez-Huamán Y, Carrasco-Loyola M. Prevalencia de caries de infancia temprana en niños menores de 6 años de edad, residentes en poblados urbano marginales de Lima Norte. Rev Estomatol Herediana. 2011; 21(2):79-86. DOI: https://doi.org/10.20453/reh. v21i 2.237

22. Villanueva-Velásquez V, Berbesí-Mendoza S, Jiménez-Valenzuela G, Báez-Quintero L. Evaluación del estado de salud oral y de conocimientos, actitudes y prácticas de escolares. Revista Nacional de Odontología. 2014; 10(19):23-30.

23. Rodríguez A, Alfonso K, Bonet M. Prácticas de salud bucal y características sociodemográficas. Rev Cubana Hig. Epidemiol. 2008; 46(3):1-15.

24. Fejerskov O. Changing paradigms in concepts on dental caries: Consequences for oral health care. Caries Res. 2004;38(3):182-91. DOI: 10.1159/000077753

25. Gao J, Ruan J, Zhao L, Zhou H, Huang R, Tian J. Oral health status and oral health knowledge,attitudes and behavior among rural children in Shaanxi, western
China: a cross-sectional survey. BMC Oral Health. 2014; 14:144. doi: 10.1186/1472-6831-14-144

26. Wilson A, Brega A, Campagna E, et al. Validation and impact of caregivers' oral health knowledge and behavior on children's oral health status. Pediatr Dent. 2016; 38(1): 47-54.

27. Wilson A, Mulvahil M, Tiwari T. The impact of maternal self-efficacy and oral health beliefs on early childhood caries in Latino children. Frontiers in Public Health 2017;5:228. doi: 10.3389/fpubh.2017.00228

28. Hooley M, Skouteris H, Boganin C, Satur J, Kilpatrick N. Parental influence and the development of dental caries in children aged $0-6$ years: A systematic review of the literatura. J Dent. 2012;40(11):873-85. doi: 10.1016/j.jdent.2012.07.013

29. UNESCO. Participación de las familias en la educación infantil Latinoamericana. Santiago de Chile: UNESCO; 2004.p.1-72.

30. Nobile C, Fortunato L, Bianco A, Pileggi C, Pavia M. Pattern and severity of Early Childhood caries in Southern Italy: a preschool- bases cross_sectional Study. BMC Public Health. 2014; 1:1-12.

Recibido: 21-06-2018

Aceptado: 13-12-2018 